В.Д. Карлов ${ }^{1}$, Ю.В. Кириченко ${ }^{2}$, А.Н. Артеменко ${ }^{3}$, О.В. Бесова ${ }^{1}$

${ }^{1}$ Харьковский начиональный университет Воздушных Сил имени И. Кожедуба, Харьков

${ }^{2}$ Национальный научный иентр Харьковский физико-технический институт, Харьков

${ }^{3}$ Генеральный штаб Вооруженных Сил Украины, Киев

\title{
ОСОБЕННОСТИ ФОРМИРОВАНИЯ ДИАГРАММ НАПРАВЛЕННОСТИ ПЛАЗМЕННОЙ АНТЕННЫ С АЗИМУТАЛЬНО-НЕСИММЕТРИЧНЫМИ БЕГУЩИМИ ВОЛНАМИ ТОКА
}

Теоретически и численно исследуется плазменная антенна с азимутально-несимметричной бегущей волной, работающей на гармонике с азимутальным номером $m=1$. Плазма, в которой учтень электрон атомные столкновения, предполагается холодной и изотропной. Исследуется зависимость диаграмм направленности от плотности плазмы, геометрических параметров плазменного ичилндра и частоты электрон атомных столкновений. Коэффициенты затухания, обусловленные столкновениями, находятся из дисперсионного уравнения. Найдены минимальные значения плотности плазмы, при которых нормированные диаграммы направленности являются остронаправленными и имеют главный лепесток, расположенный под нулевым углом к оси антенны. Показаны преимущества антенны с несимметричной волной с т=1 по сравнению с антенной, работающей на азимутально-симметричной моде.

Ключевые слова: плазменная антенна бегущей волны, антенна осевого излучения, линейная антенна, ограниченная плазма, несимметричные поверхностные волны в плазме, электрон атомные столкновения 8 плазме, диаграмма направленности.

\section{Введение}

В литературе широко обсуждается возможность использования ограниченной низкотемпературной плазмы в качестве антенн [1-4]. В этих работах показано, что при достаточно высокой плотности плазменный столб имеет дисперсионные свойства, близкие к металлическим антеннам. В работе [4] аналитически показано, что азимутально-несимметричная гармоника бегущей волны тока $\mathrm{c} \mathrm{m}=1$, в отличие от симметричной волны и волн $\mathrm{c} m>1$, дает излучение линейной антенны с нормированной диаграммой направленности (НДН), имеющей максимум под нулевым углом к ее оси. В этой же работе получены формулы для углового распределения излучения в дальней зоне, численный анализ которых позволяет детально проанализировать зависимость НДН от параметров антенны и плазмы.

\section{Постановка задачи}

В работе исследуются линейные антенны бегущей волны тока $\mathrm{c} \mathrm{m}=1$. Антенна представляет собой столб холодной изотропной плазмы с потерями, обусловленными электрон атомными столкновениями. В $[3,4]$ показано, что влияние достаточно тонкой цилиндрической оболочки, окружающей плазму, на НДН мало. Поэтому она в настоящей работе не учитывается. Исследуется зависимость НДН от плотности плазмы, геометрических параметров плазменного цилиндра, длины излучаемой волны. Учитываются также столкновения электронов с нейтральными атомами, чего не было сделано в [4].

\section{Несимметричные волны в плазменном столбе с учетом столкновений}

В работе рассматривается открытый цилиндрический плазменный столб радиуса а, неограниченный в продольном направлении (рис. 1).

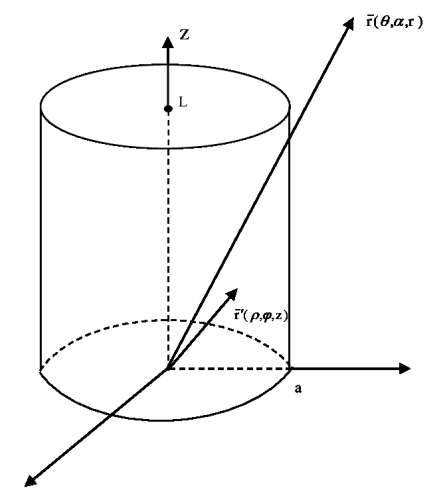

Рис. 1. Цилиндрический плазменный столб

Несимметричные с $\mathrm{m} \geq 1$ волны в таком плазменном столбе представляют собой гибридные ЕН волны, содержащие все шесть компонент электромагнитного поля. Они имеют следующую зависимость от цилиндрических координат $(\rho, \varphi, z)$, показанных на рис. 1 , и времени $\mathrm{t}$ :

$$
\overrightarrow{\mathrm{E}}, \overrightarrow{\mathrm{H}}=\overrightarrow{\mathrm{E}}(\rho), \overrightarrow{\mathrm{H}}(\rho) \exp \left(\mathrm{i}\left(\mathrm{k}_{\mathrm{z}} \mathrm{z}+\mathrm{m} \phi-\omega \mathrm{t}\right)\right),
$$

где $\mathrm{k}_{\mathrm{z}}$ - постоянная распространения, $\mathrm{m}$ - номер азимутальной гармоники.

Компоненты поля (1) в цилиндрических координатах выражаются через аксиальные составляю- 
щие электрического и магнитного полей с помощью хорошо известных формул, которые приведены, например, в [5]. Сшивая тангенциальные составляющие электрического и магнитного полей на границе плазма - вакуум, получаем следующее дисперсионное уравнение:

$$
\begin{array}{r}
\left\{\left(\kappa_{0} \mathrm{a}\right) \mathrm{K}_{\mathrm{m}}\left(\kappa_{0} \mathrm{a}\right) \mathrm{I}_{\mathrm{m}}^{\prime}(\kappa \mathrm{a})-(\kappa \mathrm{a}) \mathrm{K}_{\mathrm{m}}^{\prime}\left(\kappa_{0} \mathrm{a}\right) \mathrm{I}_{\mathrm{m}}(\kappa \mathrm{a})\right\} \times \\
\times\left\{\varepsilon_{\mathrm{p}} \cdot\left(\kappa_{0} \mathrm{a}\right) \mathrm{K}_{\mathrm{m}}\left(\kappa_{0} \mathrm{a}\right) \mathrm{I}_{\mathrm{m}}^{\prime}(\kappa \mathrm{a})-(\kappa \mathrm{a}) \mathrm{K}_{\mathrm{m}}^{\prime}\left(\kappa_{0} \mathrm{a}\right) \mathrm{I}_{\mathrm{m}}(\kappa \mathrm{a})\right\}= \\
=\left\{\frac{\mathrm{mk}_{\mathrm{z}} \mathrm{k}\left(1-\varepsilon_{\mathrm{p}}\right)}{\kappa \kappa_{0}}\right\} \mathrm{K}_{\mathrm{m}}^{2}\left(\kappa_{0} \mathrm{a}\right) \mathrm{I}_{\mathrm{m}}^{2}(\kappa \mathrm{a}) \\
\text { где } \quad \kappa_{0}=\sqrt{\mathrm{k}_{\mathrm{z}}^{2}-\mathrm{k}^{2}}, \quad \kappa=\sqrt{\mathrm{k}_{\mathrm{z}}^{2}-\mathrm{k}^{2} \varepsilon_{\mathrm{p}}}
\end{array}
$$

$\mathrm{k}^{2}=\omega^{2} / \mathrm{c}^{2}, \quad \mathrm{I}_{\mathrm{m}}(\mathrm{x}), \mathrm{K}_{\mathrm{m}}(\mathrm{x})$ - модифицированные функции Бесселя, $\mathrm{I}_{\mathrm{m}}^{\prime}(\mathrm{x}), \mathrm{K}_{\mathrm{m}}^{\prime}(\mathrm{x})$ - их производные

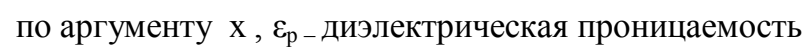
холодной плазмы с учетом электрон атомных столкновений

$$
\begin{gathered}
\varepsilon_{\mathrm{p}}=1-\frac{\omega_{\mathrm{p}}^{2}}{\omega(\omega+\mathrm{i} v)}=\varepsilon_{\mathrm{p}}^{\prime}+\mathrm{i} \varepsilon_{\mathrm{p}}^{\prime \prime}, \\
\varepsilon_{\mathrm{p}}^{\prime}=1-\omega_{\mathrm{p}}^{2} /\left(\omega^{2}+v^{2}\right), \quad \varepsilon_{\mathrm{p}}^{\prime \prime}=\omega_{\mathrm{p}}^{2} v /\left(\omega\left(\omega^{2}+v^{2}\right)\right) .
\end{gathered}
$$

где $v$ - частота столкновений электронов с атомами, $\omega_{\mathrm{p}}=\sqrt{4 \pi \mathrm{n}_{\mathrm{e}} \mathrm{e}^{2} / \mathrm{m}_{\mathrm{e}}}$ - плазменная частота, $\mathrm{n}_{\mathrm{e}}$ плотность электронов плазмы в см$^{-3}$, е и $\mathrm{m}_{\mathrm{e}}$ - заряд и масса электрона.

Полагая $\mathrm{m}=0$ в уравнении (2), получаем два дисперсионные уравнения для симметричных Н- и Е- волн, из которых только последняя может существовать в плазменном столбе. Дисперсионное уравнение для нее имеет вид

$$
\left\{\varepsilon_{\mathrm{p}}\left(\kappa_{0} \mathrm{a}\right) \mathrm{K}_{0}\left(\kappa_{0} \mathrm{a}\right) \mathrm{I}_{1}(\kappa \mathrm{a})+(\kappa \mathrm{a}) \mathrm{K}_{1}\left(\kappa_{0} \mathrm{a}\right) \mathrm{I}_{0}(\kappa \mathrm{a})\right\}=0 .
$$

В (6) учтены соотношения $\mathrm{I}_{0}^{\prime}(\mathrm{x})=\mathrm{I}_{1}(\mathrm{x})$, $\mathrm{K}_{0}^{\prime}(\mathrm{x})=-\mathrm{K}_{1}(\mathrm{x})$. При выводе уравнений $(2,6)$ учтено, что аксиальные составляющие полей (1) определяются соотношениями $\mathrm{E}_{\mathrm{z}}(\rho)=\mathrm{AI}_{\mathrm{m}}(\kappa \rho)$, $\mathrm{H}_{\mathrm{z}}(\rho)=\mathrm{BI}_{\mathrm{m}}(\kappa \rho)$, где А, В постоянные.

Столкновения электронов с атомами вызывает затухание волн, которое проявляется в том, что волновой вектор $\mathrm{k}_{\mathrm{z}}$ в (1) становится комплексным $\mathrm{k}_{\mathrm{z}}=\mathrm{k}_{\mathrm{z}}^{\prime}+\mathrm{i} \mathrm{k}_{\mathrm{z}}^{\prime \prime}$. Соответственно аргументы функций $\mathrm{I}_{\mathrm{m}}(\mathrm{x}), \mathrm{K}_{\mathrm{m}}(\mathrm{x})$ также будут комплексными. Уравнение (2) выведено при следующих условиях: $\omega<\omega_{\mathrm{p}}$, $\mathrm{k}_{\mathrm{z}}^{\prime}>\mathrm{k}$. В настоящей работе предполагается, что затухание волн, обусловленное столкновениями, мало. Поэтому малыми будут следующие параметры:

$$
v / \omega<<1, \mathrm{k}_{\mathrm{z}}{ }^{\prime} / \mathrm{k}_{\mathrm{z}}{ }^{\prime}<<1 .
$$

Ограничиваясь линейными по параметрам (7) членами, получим из (3) формулы

$$
\begin{gathered}
\kappa_{0}=\kappa_{0}^{\prime}+\mathrm{i} \kappa_{0}^{\prime \prime}, \kappa_{0}^{\prime}=\sqrt{\mathrm{k}_{\mathrm{z}}^{\prime 2}-\mathrm{k}^{2}}, \kappa_{0}^{\prime \prime}=\mathrm{k}_{\mathrm{z}}^{\prime} \mathrm{k}_{\mathrm{z}}^{\prime \prime} / \kappa_{0}^{\prime} \\
\kappa=\kappa^{\prime}+\mathrm{i} \kappa^{\prime \prime}, \kappa^{\prime}=\sqrt{\kappa_{0}^{\prime}+\omega_{\mathrm{p}}^{2} / \mathrm{c}^{2}} \\
\kappa^{\prime \prime}=\left(\mathrm{k}_{\mathrm{z}}^{\prime} \mathrm{k}_{\mathrm{z}}^{\prime \prime}-\mathrm{k}^{2} \omega_{\mathrm{p}}^{2} v /\left(2 \omega^{3}\right)\right) / \kappa^{\prime} . \\
\varepsilon_{\mathrm{p}}^{\prime}=1-\frac{\omega_{\mathrm{p}}^{2}}{\omega^{2}}, . \quad \varepsilon_{\mathrm{p}}^{\prime \prime}=\frac{\omega_{\mathrm{p}}^{2} v}{\omega^{3}}
\end{gathered}
$$

Из условий (7) следуют также неравенства

$$
\frac{\kappa_{0}^{\prime \prime}}{\kappa_{0}^{\prime}}<<1, \frac{\kappa^{\prime \prime}}{\kappa^{\prime}}<<1, \frac{\varepsilon_{\mathrm{p}}^{\prime \prime}}{\left|\varepsilon_{\mathrm{p}}^{\prime}\right|}<<1 .
$$

Условия (8) означают, что мнимые части аргументов функций $\mathrm{I}_{\mathrm{m}}(\mathrm{x}), \mathrm{K}_{\mathrm{m}}(\mathrm{x})$ малы по сравнению с действительными. Это позволяет разложить уравнение (2) по малым мнимым частям аргументов, выделить в нем действительную и мнимую части и получить два уравнения. Первое из них получается заменой $\kappa_{0} \rightarrow \kappa_{0}^{\prime}, \kappa \rightarrow \kappa^{\prime}, \varepsilon_{\mathrm{p}} \rightarrow \varepsilon_{\mathrm{p}}^{\prime}$ в (2) и является дисперсионным уравнением, решение которого дает зависимость $\omega=\omega\left(\mathrm{k}_{\mathrm{z}}^{\prime}\right)$. Второе уравнение дает такое выражение для коэффициента затухания $\mathrm{k}_{\mathrm{z}}$ :

$a k_{z}^{\prime \prime}=\frac{v}{\omega} \frac{\kappa_{0}^{\prime}}{k_{z}^{\prime}} \frac{t_{2} t_{10}+t_{3}\left(t_{7}-t_{9} y_{0}\right)-y_{0} t_{5} t_{6}}{t_{1} t_{10}-t_{3} t_{8}-t_{6} t_{4}-\left(t_{3} t_{9}+t_{6} t_{5}\right) \kappa_{0}^{\prime} / \kappa^{\prime}}$

где

$$
\begin{aligned}
& \mathrm{t}_{1}=\kappa_{0}^{\prime} / \kappa^{\prime} \cdot\left(\mathrm{K}_{\mathrm{m}} \mathrm{I}_{\mathrm{m}}^{\prime}-\mathrm{K}_{\mathrm{m}} \mathrm{I}_{\mathrm{m}} / \mathrm{y}_{1}\right)+ \\
& +\left(\left(\mathrm{x}_{1} /\left(a \mathrm{k}_{\mathrm{z}}^{\prime}\right)^{2}-1 / \mathrm{x}_{1}\right) \mathrm{K}_{\mathrm{m}} \mathrm{K}_{\mathrm{m}}^{\prime}\right) \mathrm{I}_{\mathrm{m}},
\end{aligned}
$$

$\mathrm{t}_{2}=\left(\mathrm{K}_{\mathrm{m}} \mathrm{I}_{\mathrm{m}}^{\prime}-\frac{1}{\mathrm{y}_{1}} \mathrm{~K}_{\mathrm{m}} \mathrm{I}_{\mathrm{m}}\right) \mathrm{y}_{0}+\mathrm{K}_{\mathrm{m}} \mathrm{I}_{\mathrm{m}}$,

$\mathrm{t}_{3}=\mathrm{x}_{1} \mathrm{~K}_{\mathrm{m}} \mathrm{I}_{\mathrm{m}}^{\prime}-\mathrm{y}_{1} \mathrm{~K}_{\mathrm{m}}^{\prime} \mathrm{I}_{\mathrm{m}}$,

$\mathrm{t}_{4}=\left(\mathrm{K}_{\mathrm{m}}+\mathrm{x}_{1} \mathrm{~K}_{\mathrm{m}}^{\prime}\right) \mathrm{I}_{\mathrm{m}}^{\prime}-\mathrm{y}_{1} \mathrm{~K}_{\mathrm{m}}^{\prime \prime} \mathrm{I}_{\mathrm{m}}$,

$\mathrm{t}_{5}=\mathrm{x}_{1} \mathrm{~K}_{\mathrm{m}} \mathrm{I}_{\mathrm{m}}^{\prime \prime}-\mathrm{K}_{\mathrm{m}}^{\prime}\left(\mathrm{y}_{1} \mathrm{I}_{\mathrm{m}}^{\prime}+\mathrm{I}_{\mathrm{m}}\right)$,

$\mathrm{t}_{6}=\varepsilon_{\mathrm{p}}^{\prime} \mathrm{x}_{1} \mathrm{~K}_{\mathrm{m}} \mathrm{I}_{\mathrm{m}}^{\prime}-\mathrm{y}_{1} \mathrm{~K}_{\mathrm{m}}^{\prime} \mathrm{I}_{\mathrm{m}}, \quad \mathrm{t}_{7}=\frac{\omega_{\mathrm{p}}^{2}}{\omega^{2}} \mathrm{x}_{1} \mathrm{~K}_{\mathrm{m}} \mathrm{I}_{\mathrm{m}}^{\prime}$

$\mathrm{t}_{8}=\varepsilon_{\mathrm{p}}^{\prime} \mathrm{I}_{\mathrm{m}}^{\prime}\left(\mathrm{K}_{\mathrm{m}}+\mathrm{x}_{1} \mathrm{~K}_{\mathrm{m}}^{\prime}\right)-\mathrm{y}_{1} \mathrm{~K}_{\mathrm{m}}^{\prime \prime} \mathrm{I}_{\mathrm{m}}$

$\mathrm{t}_{9}=\varepsilon_{\mathrm{p}}^{\prime} \mathrm{x}_{1} \mathrm{~K}_{\mathrm{m}} \mathrm{I}_{\mathrm{m}}^{\prime \prime}-\mathrm{K}_{\mathrm{m}}^{\prime}\left(\mathrm{y}_{1} \mathrm{I}_{\mathrm{m}}^{\prime}+\mathrm{I}_{\mathrm{m}}\right)$,

$\mathrm{t}_{10}=2\left(\frac{\mathrm{m}(\mathrm{ak})\left(\mathrm{ak}_{\mathrm{z}}^{\prime}\right) \omega_{\mathrm{p}}^{2}}{\mathrm{x}_{1} \mathrm{y}_{1} \omega^{2}}\right)^{2} \mathrm{~K}_{\mathrm{m}} \mathrm{I}_{\mathrm{m}}, \mathrm{y}_{0}=\frac{(\mathrm{ak})^{2} \omega_{\mathrm{p}}^{2}}{2 \omega^{2} \mathrm{y}_{1}}$

В формулах для $\mathrm{t}_{\mathrm{i}}(\mathrm{i}=1 \ldots 10)$ функция $\mathrm{K}_{\mathrm{m}}$ зависит от аргумента $\mathrm{x}_{1}=\mathrm{a} \kappa_{0}^{\prime}$, а функция $\mathrm{I}_{\mathrm{m}}$ от $\mathrm{y}_{1}=\mathrm{a \kappa}$ '. 
Эти аргументы не указаны для краткости изложения. Аналогично из уравнения (6) получается дисперсионная зависимость $\omega=\omega\left(\mathrm{k}_{\mathrm{Z}}^{\prime}\right)$ и формула для коэффициента затухания $\mathrm{k}_{\mathrm{z}}$ д для симметричной Е-волны.

$$
\begin{gathered}
\mathrm{ak}_{\mathrm{z}}^{\prime \prime}=\frac{v}{\omega} \frac{\kappa_{0}^{\prime}}{\mathrm{k}_{\mathrm{z}}^{\prime}} \frac{\mathrm{y}_{0} \mathrm{~s}_{2}-\mathrm{s}_{3}}{\mathrm{~s}_{1}+\mathrm{s}_{2} \kappa_{0}^{\prime} / \kappa^{\prime}}, \\
\text { где } \mathrm{s}_{1}=\varepsilon_{\mathrm{p}}^{\prime} \mathrm{I}_{0}^{\prime}\left(\mathrm{K}_{0}+\mathrm{x}_{1} \mathrm{~K}_{0}^{\prime}\right)-\mathrm{y}_{1} \mathrm{~K}_{0}^{\prime \prime} \mathrm{I}_{0}, \\
\mathrm{~s}_{2}=\varepsilon_{\mathrm{p}}^{\prime} \mathrm{x}_{1} \mathrm{~K}_{0} \mathrm{I}_{0}^{\prime \prime}-\mathrm{K}_{0}^{\prime}\left(\mathrm{y}_{1} \mathrm{I}_{0}^{\prime}+\mathrm{I}_{0}\right), \mathrm{s}_{3}=\mathrm{x}_{1} \frac{\omega_{\mathrm{p}}^{2}}{\omega^{2}} \mathrm{~K}_{0} \mathrm{I}_{0}^{\prime}
\end{gathered}
$$

\section{Излучение плазменной антенны}

Для нахождения углового распределения излучения плазменного столба исходим из формулы для запаздывающего векторного потенциала

$$
\overrightarrow{\mathrm{A}}(\overrightarrow{\mathrm{r}}, \mathrm{t})=\frac{1}{\mathrm{c}} \int \mathrm{d}^{3} \mathrm{r}^{\prime} \frac{\overrightarrow{\mathrm{j}}\left(\overrightarrow{\mathrm{r}}^{\prime}, \mathrm{t}^{\prime}\right)}{\mathrm{R}},
$$

где $\mathrm{R}=\left|\overrightarrow{\mathrm{r}}-\overrightarrow{\mathrm{r}}^{\prime}\right|, \mathrm{t}^{\prime}=\mathrm{t}-\frac{1}{\mathrm{c}} \mathrm{R}, \overrightarrow{\mathrm{j}}\left(\overrightarrow{\mathrm{r}}^{\prime}, \mathrm{t}^{\prime}\right)=\sigma \overrightarrow{\mathrm{E}}\left(\overrightarrow{\mathrm{t}}^{\prime}, \mathrm{t}^{\prime}\right)$.

В формуле (11) переменная интегрирования $\vec{r}^{\prime}(\rho, \phi, z)$ принадлежит плазменному цилиндру, a $\overrightarrow{\mathrm{r}}(\alpha, \theta, \mathrm{r})$ - точка наблюдения (рис.1). В формуле (12) $\sigma$ - проводимость плазмы, $\overrightarrow{\mathrm{E}}\left(\overrightarrow{\mathrm{r}}^{\prime}, \mathrm{t}^{\prime}\right)$ - поле волны в плазменном столбе. Магнитное поле $\overrightarrow{\mathrm{H}}(\overrightarrow{\mathrm{r}}, \mathrm{t})$ излучения в дальней зоне получается из (11). Формула для него имеет вид

$$
\begin{gathered}
\overrightarrow{\mathrm{H}}(\overrightarrow{\mathrm{r}}, \mathrm{t})=\frac{\mathrm{ik} \sigma}{\mathrm{c}} \cdot \frac{\exp (\mathrm{i}(\mathrm{kr}-\omega \mathrm{t}))}{\mathrm{r}} \times, \\
\times \int \mathrm{d}^{3} \mathrm{r}^{\prime}\left[\overrightarrow{\mathrm{e}}_{\mathrm{r}} \times \overrightarrow{\mathrm{E}}\left(\overrightarrow{\mathrm{r}}^{\prime}\right)\right] \exp \left(-\mathrm{ik}\left(\overrightarrow{\mathrm{r}}^{\prime} \overrightarrow{\mathrm{e}}_{\mathrm{r}}\right)\right),
\end{gathered}
$$

где $\overrightarrow{\mathrm{e}}_{\mathrm{r}}=\overrightarrow{\mathrm{r}} / \mathrm{r}$. Вычисляя вектор Пойнтинга в дальней зоне с помощью соотношения (13), находим угловое распределение излучения.

Для несимметричных волн в антенне угловое распределение определяется в нулевом порядке по параметрам (8) формулой:

$$
\begin{aligned}
& \frac{\mathrm{dP}}{\mathrm{d} \Omega}=|\mathrm{A}|^{2} \frac{\mathrm{L}^{2} \mathrm{ck}^{4}}{32 \pi}\left(\frac{\omega_{\mathrm{p}}}{\omega}\right)^{4} \exp \left(-2 \mathrm{k}_{\mathrm{z}}^{\prime \prime} \mathrm{L}\right) \Psi_{1}(\theta) \Psi_{2}(\theta), \\
& \text { где } \quad \Psi_{1}(\theta)=\frac{\sin ^{2} \mathrm{X}(\theta)+\mathrm{sh}^{2}\left(0.5 \mathrm{Lk}_{\mathrm{z}}^{\prime \prime}\right)}{\mathrm{X}^{2}(\theta)+\left(0.5 \mathrm{Lk}_{\mathrm{z}}^{\prime \prime}\right)^{2}}, \\
& \mathrm{X}(\theta)=0.5 \mathrm{~kL}(\xi-\cos \theta) \\
& \xi=\mathrm{ck}_{\mathrm{z}}^{\prime} / \omega\left(\mathrm{k}_{\mathrm{z}}^{\prime}\right)-\text { замедление волны в антенне, } \\
& \Psi_{2}(\theta)=\cos ^{2} \theta\left(\mathrm{f}_{1}-\mathrm{mf}_{2}\right)^{2}+ \\
& +\mathrm{f}_{3}\left(\sin ^{2} \theta \mathrm{f}_{3}+\sin 2 \theta\left(\mathrm{f}_{1}-\mathrm{mf}_{2}\right)\right)+\left(\mathrm{mf}_{4}-\mathrm{f}_{5}\right)^{2}
\end{aligned}
$$

$$
\begin{aligned}
& \mathrm{f}_{1}=\frac{1}{\kappa^{\prime 2}} \int_{0}^{\mathrm{a}} \mathrm{d} \rho \rho J_{\mathrm{m}}^{\prime}(\tilde{\rho})\left(-\mathrm{k}_{\mathrm{z}}^{\prime} \kappa \mathrm{I}_{\mathrm{m}}^{\prime}\left(\kappa^{\prime} \rho\right)+\frac{\mathrm{mk}}{\rho} \mathrm{QI} \mathrm{I}_{\mathrm{m}}\left(\kappa^{\prime} \rho\right)\right) ; \\
& \mathrm{f}_{2}=\frac{1}{\kappa^{\prime 2}} \int_{0}^{\mathrm{a}} \mathrm{d} \rho \rho \frac{\mathrm{J}_{\mathrm{m}}(\tilde{\rho})}{\tilde{\rho}}\left(\frac{\mathrm{mk}_{\mathrm{z}}^{\prime}}{\rho} \mathrm{I}_{\mathrm{m}}\left(\kappa^{\prime} \rho\right)-\mathrm{Qk} \kappa^{\prime} \mathrm{I}_{\mathrm{m}}^{\prime}\left(\kappa^{\prime} \rho\right)\right) ; \\
& \mathrm{f}_{3}=\int_{0}^{\mathrm{a}} \mathrm{d} \rho \rho \mathrm{J}_{\mathrm{m}}(\tilde{\rho}) \mathrm{I}_{\mathrm{m}}\left(\kappa^{\prime} \rho\right) \\
& \mathrm{f}_{4}=\frac{1}{\kappa^{\prime 2}} \int_{0}^{\mathrm{a}} \mathrm{d} \rho \rho \frac{\mathrm{J}_{\mathrm{m}}(\tilde{\rho})}{\tilde{\rho}}\left(-\mathrm{k}_{\mathrm{z}}^{\prime} \kappa^{\prime} \mathrm{I}_{\mathrm{m}}^{\prime}\left(\kappa^{\prime} \rho\right)+\mathrm{Q} \frac{\mathrm{mk}}{\rho} \mathrm{I}_{\mathrm{m}}\left(\kappa^{\prime} \rho\right)\right) \\
& \mathrm{f}_{5}=\frac{1}{\kappa^{\prime 2}} \int_{0}^{\mathrm{a}} \mathrm{d} \rho \rho J_{\mathrm{m}}^{\prime}(\tilde{\rho})\left(\frac{\mathrm{mk}_{\mathrm{z}}^{\prime}}{\rho} \mathrm{I}_{\mathrm{m}}\left(\kappa^{\prime} \rho\right)-\mathrm{Qk} \kappa^{\prime} \mathrm{I}_{\mathrm{m}}^{\prime}\left(\kappa^{\prime} \rho\right)\right)
\end{aligned}
$$

В формулах (17-21) приняты следующие обозначения: $\tilde{\rho}=\mathrm{k} \rho \sin \theta, \mathrm{J}_{\mathrm{m}}(\mathrm{x})$ - функция Бесселя, $\mathrm{J}_{\mathrm{m}}^{\prime}(\mathrm{x})$ - ее производная по аргументу $\mathrm{x}$, $\mathrm{Q}=-\mathrm{iH}_{\mathrm{z}}(\rho) / \mathrm{E}_{\mathrm{z}}(\rho)=-\mathrm{iB} / \mathrm{A}$. Величина $\mathrm{Q}$ определяется формулой

$$
\frac{\mathrm{Q}}{\mathrm{a}}=\kappa^{\prime} \kappa_{0}^{\prime} \frac{\kappa^{\prime} \mathrm{K}_{\mathrm{m}}^{\prime}\left(\kappa_{0}^{\prime} \mathrm{a}\right) \mathrm{I}_{\mathrm{m}}\left(\kappa^{\prime} \mathrm{a}\right)-\varepsilon_{\mathrm{p}}^{\prime} \kappa_{0}^{\prime} \mathrm{K}_{\mathrm{m}}\left(\kappa_{0}^{\prime} \mathrm{a}\right) \mathrm{I}_{\mathrm{m}}^{\prime}\left(\kappa^{\prime} \mathrm{a}\right)}{\mathrm{mk}_{\mathrm{z}}^{\prime} \mathrm{k}\left(1-\varepsilon_{\mathrm{p}}^{\prime}\right) \mathrm{K}_{\mathrm{m}}\left(\kappa_{0}^{\prime} \mathrm{a}\right) \mathrm{I}_{\mathrm{m}}\left(\kappa^{\prime} \mathrm{a}\right)} .
$$

Отметим, что при малом замедлении $\xi$ и достаточно больших длинах волн излучения $\lambda$, когда выполняются условия

$$
\xi-1<<1, \mathrm{a}<<\lambda,
$$

справедливо соотношение $\mathrm{Q} \approx-1$, Это означает, что компоненты поля волны $\mathrm{E}_{\mathrm{z}}(\rho)$ и $\mathrm{H}_{\mathrm{z}}(\rho)$ близки по амплитуде и сдвинуты по фазе на $\pi / 2$, так как

$$
\mathrm{H}_{\mathrm{z}}(\rho) / \mathrm{E}_{\mathrm{z}}(\rho) \approx-\mathrm{i} .
$$

Функция $\Psi_{1}(\theta)$ (15) описывает угловое распределение излучения однородной по поперечному сечению линейной антенны [6, 7]. Она зависит от материала антенны только посредством зависимости от замедления $\xi$. Функция $\Psi_{2}(\theta)(16)$ определяется действительной частью диэлектрической проницаемости $\varepsilon_{\mathrm{p}}^{\prime}$, то есть внутренней структурой плазменной антенны.

Для углового распределения в случае симметричных волн получается в нулевом порядке по параметрам (8) соотношение

$$
\begin{aligned}
& \frac{\mathrm{dP}}{\mathrm{d} \Omega}=|\mathrm{A}|^{2} \frac{\mathrm{L}^{2} \mathrm{ck}^{4}}{32 \pi}\left(\frac{\omega_{\mathrm{p}}}{\omega}\right)^{4} \times \\
& \times \exp \left(-2 \mathrm{k}_{\mathrm{z}}^{\prime \prime} \mathrm{L}\right) \Psi_{1}(\theta) \Psi_{3}(\theta), \\
& \Psi_{3}(\theta)=\left(\cos \theta \mathrm{f}_{6}+\sin \theta \mathrm{f}_{5}\right)^{2}, \\
& \mathrm{f}_{6}=\frac{\mathrm{k}_{\mathrm{z}}^{\prime}}{\kappa^{\prime}} \int_{0}^{\mathrm{a}} \mathrm{d} \rho \rho \mathrm{J}_{1}(\tilde{\rho}) \mathrm{I}_{1}\left(\kappa^{\prime} \rho\right),
\end{aligned}
$$




$$
\mathrm{f}_{7}=\int_{0}^{\mathrm{a}} \mathrm{d} \rho \rho \mathrm{J}_{0}(\tilde{\rho}) \mathrm{I}_{0}\left(\kappa^{\prime} \rho\right) .
$$

Для симметричных волн внутренняя структура плазменной антенны описывается функцией $\Psi_{3}(\theta)$. Из формул (22-24) следует, что излучение под углом $\theta=0$ отсутствует. Соотношения $(14,22)$ позволяют найти нормированные диаграммы направленности .

\section{Результаты вычислений}

В работе [4] выполнен аналитический анализ диаграмм направленности при условиях (22). Показано, что угловая зависимость НДН антенны с азимутально-симметричной волной имеет один хорошо выраженный лепесток с низким уровнем боковых лепестков. При этом максимум НДН приходится на угол $\theta$, отличный от нуля. Показано также, что при тех же условиях волна $\mathrm{c} m=1$ дает излучение с однолепестковой НДН, имеющей максимум при $\theta=0$. В настоящей работе проведен численный анализ НДН для гармоник $\mathrm{c} \quad \mathrm{m}=0,1$ в зависимости от замедления волны $\xi$, геометрических параметров антенны, длины излучаемой волны и частоты электрон атомных столкновений.

Угловое распределение излучения сильно зависит от замедления волны в антенне $\xi$, которое в свою очередь определяется плотностью плазмы $\mathrm{n}_{\mathrm{e}}$. При этом оказывается, что при больших $\xi$, когда условия (22) не выполняются, НДН для гармоник с $\mathrm{m}=0,1$ имеют много лепестков, среди которых нельзя выделить один главный. Для волн $\mathrm{c} \mathrm{m}=0$ и $\mathrm{m}=1$ существуют свои характерные значения плотности плазмы $\mathrm{n}_{\mathrm{e} 0}$ такие, что при $\mathrm{n}_{\mathrm{e}} \geq \mathrm{n}_{\mathrm{e} 0}$ диаграммы направленности имеют один хорошо выраженный лепесток с малым уровнем боковых лепестков. Поэтому для анализа НДН важно знать зависимость замедления волны от плотности плазмы.

Вычисления с параметрами $\mathrm{a}=1 \mathrm{~cm}, \mathrm{~L}=100 \mathrm{~cm}$, $\lambda==10 \mathrm{~cm}$., $\mathrm{m}=1$ дали следующие результаты. На рис.2 представлена зависимость величины $\lg (\xi-1)$ от $\lg \left(\mathrm{n}_{\mathrm{e}}\right)$.

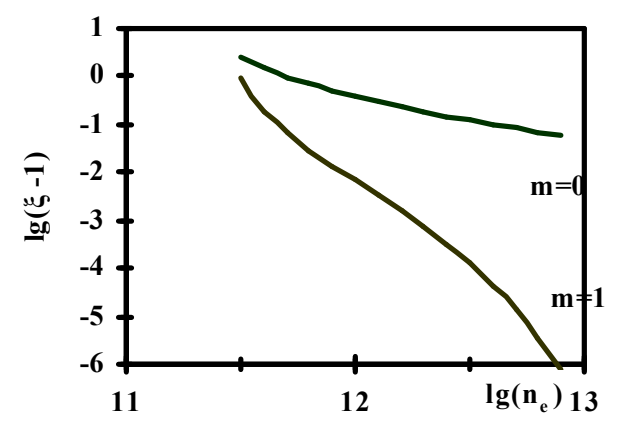

Рис. 2. Зависимость замедления $\xi$ волны в плазменной антенне от плотности плазмы для гармоник $\mathrm{c} \mathrm{m}=0,1$
Видно, что замедление $\xi$ волны $\mathrm{c} \mathrm{m}=1$ намного быстрее приближается к 1 , чем замедление азимутально симметричной волны. Поэтому антенна с гармоникой $\mathrm{m}=1$ дает излучение $\mathrm{c}$ хорошо выраженной направленностью при $\mathrm{n}_{\mathrm{e}}$, меньших, чем антенна с гармоникой $\mathrm{m}=0$. Другими словами гармоника с $\mathrm{m}=1$ при увеличении $\mathrm{n}_{\mathrm{e}}$ намного быстрее приближается по своим дисперсионным свойствам к металлической антенне, чем антенна с $\mathrm{m}=0$. Из рис. 2 видно, при $\mathrm{n}_{\mathrm{e}} \approx 8.10^{12} \mathrm{~cm}^{-3}$ фазовая скорость несимметричной волны $\mathrm{c} \mathrm{m}=1$ отличается от скорости света в пятом знаке после запятой. Примерно такое же отличие от скорости света имеют волны в металлической антенне в дециметровом диапазоне излучения.

На рис. 3 - 5 представлены НДН для антенны с приведенными выше параметрами $\mathrm{a}, \mathrm{L}, \lambda$ и $\mathrm{c}$ плотностью плазмы $\lg \left(\mathrm{n}_{\mathrm{e}}\right)=11.8$ для следующих значений параметра $v / \omega$, характеризующего затухание волны в плазме: $v / \omega=0,0,1$ и 0,4 соответственно.

Видно, что излучение направлено вдоль оси антенны и имеет очень малый уровень боковых лепестков. Главный лепесток при $v / \omega=0$ является узким и имеет полуширину равную примерно $13^{0}$.

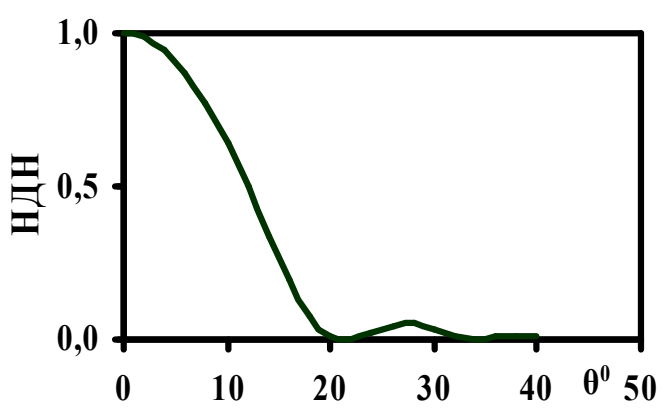

Рис. 3. Нормированная диаграмма направленности для $\mathrm{m}=1, \mathrm{a}=1 \mathrm{~cm}, \mathrm{~L}=100 \mathrm{~cm}, \lambda=10 \mathrm{~cm}$,

$$
\lg \left(\mathrm{n}_{\mathrm{e}}\right)=11.8, v / \omega=0
$$

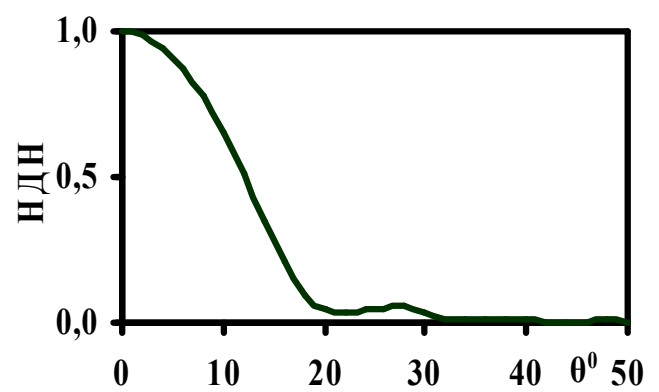

Рис. 4. Нормированная диаграмма направленности для $\mathrm{m}=1, \mathrm{a}=1 \mathrm{~cm}, \mathrm{~L}=100 \mathrm{~cm}, \lambda=10 \mathrm{~cm}$,

$$
\lg \left(\mathrm{n}_{\mathrm{e}}\right)=11.8, v / \omega=0,1
$$




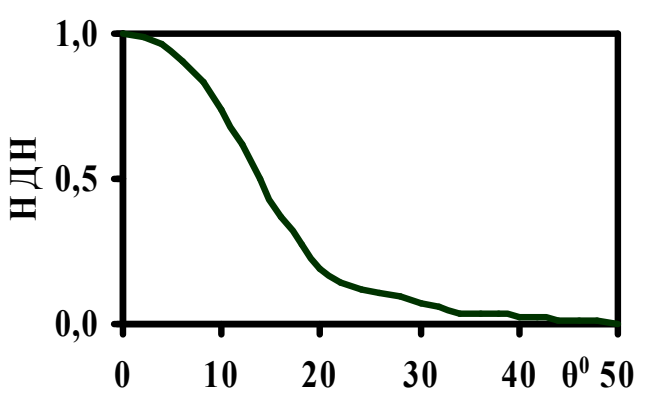

Рис. 5. Нормированная диаграмма направленности для $\mathrm{m}=1, \mathrm{a}=1 \mathrm{~cm}, \mathrm{~L}=100 \mathrm{~cm}, \lambda=10 \mathrm{~cm}$,

$$
\lg \left(\mathrm{n}_{\mathrm{e}}\right)=11.8, v / \omega=0,4
$$

В то же время излучение антенны при той же плотности плазмы с аксиально-симметричной гармоникой тока является многолепестковым с плохо выраженной направленностью.

Анализ показал, что для приведенных выше параметров и для несимметричной волны с $\mathrm{m}=1$ величина $\lg \left(\mathrm{n}_{\mathrm{e} 0}\right)=11.7$.

Это значение плотности $\mathrm{n}_{\mathrm{e} 0}$ примерно в 50 раз меньше, чем для симметричной волны. Таким образом, хорошо направленное излучение при $\mathrm{m}=1$ можно получить при значительно меньших плотностях плазмы $\mathrm{n}_{\mathrm{e}}$, чем в случае симметричной гармоники.

Из рис. 3,4,5 видно, что влияние затухания на НДН сводится к исчезновению нулей интенсивности излучения и уширению основного лепестка. Эти особенности характерны для линейных антенн бегущей волны [6, 7 - 12]. Дополнительный анализ показывает [9 - 12], что влияние параметра $v / \omega$ на плазменную антенну ослабляется с ростом плотности плазмы. В частности, увеличивается расстояние $1_{0}$ вдоль антенны, на котором волна затухает в е раз. Величина $1_{0}$ определяется соотношением $1_{0}=1 / \mathrm{k}_{\mathrm{z}}^{\prime \prime}$. При плотности $\lg \left(\mathrm{n}_{\mathrm{e}}\right)=11.8$ для $\mathrm{v} / \omega=0.1$ и для $v / \omega=0.4$ величина $1_{0}$ равна $162 \mathrm{~cm} \mathrm{и} 40 \mathrm{~cm}$ соответственно. При плотности плазмы $\lg \left(\mathrm{n}_{\mathrm{e}}\right)=11.9$ величина $1_{0}$ равна $367 \mathrm{~cm}$. при $v / \omega=0.1$, а при $v / \omega=0.41_{0}$ равно $92 \mathrm{~cm}$. То есть затухание несимметричной волны с $\mathrm{m}=1$ уменьшается с ростом плотности плазмы. Отметим также, что при заданном параметре $v / \omega$ несимметричная волна с $\mathrm{m}=1$ затухает слабее, чем симметричная. Влияние параметра $v / \omega$ на диаграммы направленности ослабляется с ростом плотности плазмы. Это влияние намного слабее для гармоники с $\mathrm{m}=1$, чем для гармоники с $\mathrm{m}=0$. Отметим, что НДН слабо зависит от плотности плазмы при $\mathrm{n}_{\mathrm{e}} \geq \mathrm{n}_{\mathrm{e} 0}$. Так при изменении $\lg \left(\mathrm{n}_{\mathrm{e}}\right)$ от 11.8 до 12.7 полуширина НДН $\Delta \theta$ изменяется от $13^{0}$ до $17^{0}$, то есть всего лишь на $4^{0}$.

Представляет также интерес поведение НДН в зависимости от длины антенны. Вычисления с па- раметрами а $=1 \mathrm{~cm}, \lambda=10 \mathrm{~cm}, \lg \left(\mathrm{n}_{\mathrm{e}}\right)=11.8$, для $\mathrm{v} / \omega=0$ показали, что при уменьшении L от 100 см до $60 \mathrm{~cm}$ полуширина НДН $\Delta \theta$ увеличивается от $13^{0}$ до $18^{\circ}$. Поэтому за счет некоторого увеличения полуширины НДН можно значительно уменьшить длину плазменной антенны. При $\mathrm{L}=20 \mathrm{~cm}$ полуширина равна $\Delta \theta=32^{0}$.

В работе [8] показано, что рабочая плотность плазмы в антенне может быть уменьшена при увеличении ее радиуса и пропорциональном увеличении длины излучаемой волны $\lambda$. Для примера, в настоящей работе были проведены вычисления для антенны с такими параметрами: $\mathrm{a}=2 \mathrm{~cm}, \mathrm{~L}=100 \mathrm{~cm}$, $\lambda=20 \mathrm{~cm}$. На рис. 6 приведены соответствующие зависимости $\lg (\xi-1)$ от $\lg \left(\mathrm{n}_{\mathrm{e}}\right)$ для $\mathrm{m}=0$ и $\mathrm{m}=1$. Видно, что при $\mathrm{m}=1$ замедление $\xi$ приближается к 1 при значительно меньших значениях плазмы, чем при $\mathrm{m}=0$. В частности, оказывается, что для такой антенны $\lg \left(\mathrm{n}_{\mathrm{e} 0}\right)=11.1$. То есть характерное значение плотности $\mathrm{n}_{\mathrm{e} 0}$, для антенны с $\mathrm{a}=2 \mathrm{~cm}$. оказывается в 4 раза меньше, чем для антенны с $а=1 \mathrm{~cm}$. Зависимость НДН от плотности плазмы слаба и в этом случае. Однако полуширина $\Delta \theta$ главного лепестка больше примерно на $7^{0}$, чем для антенны с $\mathrm{a}=1 \mathrm{~cm}$. Уровень боковых лепестков мал. Для уменьшения $\Delta \theta$ следует увеличивать длину антенны L. Однако значительное увеличение L до значений больших $100 \mathrm{~cm} \mathrm{вряд} \mathrm{ли} \mathrm{целесообразно} \mathrm{с} \mathrm{практической} \mathrm{точки}$ зрения.

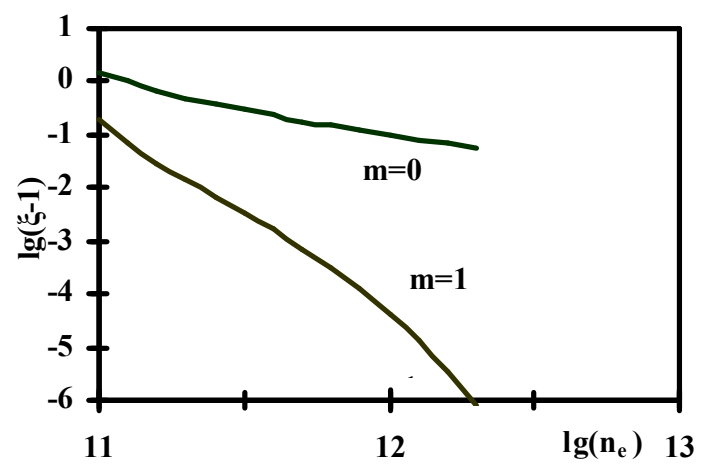

Рис. 6. Зависимости $\lg (\xi-1)$ от $\lg \left(\mathrm{n}_{\mathrm{e}}\right)$ для $\mathrm{m}=0$ и $\mathrm{m}=1$

Приведенные в работе исследования линейной плазменной антенны, работающей на гармонике волны тока $\mathrm{m}=1$, показали следующие преимущества ее по сравнению с антенной, у которой волна тока является азимутально-симметричной. Рабочая плотность плазмы антенны с $\mathrm{m}=1$ значительно меньше, чем антенны с m=0. По своим дисперсионным свойствам она намного ближе к металлической антенне, чем антенна с $\mathrm{m}=0$. Антенна с $\mathrm{m}=1$ излучает в строго осевом направлении. Для такой антенны НДН слабее зависит от потерь энергии в плазме, обусловленных столкновениями электронов с атомами, чем для антенн с m=0. 


\section{Выводы}

Теоретически и численно исследуются диаграммы направленности плазменной антенны с азимутально-несимметричной бегущей волной, работающей на гармонике $\mathrm{c} \mathrm{m}=1$. Плазма, в которой учтены электрон атомные столкновения, предполагается холодной и изотропной. Исследуется зависимость диаграмм направленности от плотности плазмы, геометрических параметров плазменного цилиндра и частоты электрон атомных столкновений. Показаны преимущества антенны с несимметричной волной, у которой $\mathrm{m}=1$, по сравнению с антенной, работающей на азимутально-симметричной волне. В частности, рабочая плотность плазмы антенны с $\mathrm{m}=1$ значительно меньше, чем антенны $\mathrm{c} \mathrm{m}=0$. По своим дисперсионным свойствам она намного ближе к металлической антенне, чем антенна с $\mathrm{m}=0$. Антенна с $\mathrm{m}=1$ излучает в строго осевом направлении. Для такой антенны НДН слабее зависит от потерь энергии в плазме, обусловленных столкновениями электронов с атомами, чем для антенн с $\mathrm{m}=0$.

\section{Список літератури}

1. Истомин Е.Н. Плазменный несимметричный вибратор с возбуждением поверхностной волной/ Истомин Е.Н., Карфидов Д.М., Минаев И.М, Рухадзе А.А. // Физика плазмы - 2006. - m. 32, №5 - C.423-435.

2. Allexeff I. Experimental and theoretical results with plasma antennas/Allexeff I., Anderson T., Parameswaran S. et al // IEEE Transaction on plasma science. - 2006.- Vol. 34, No. 2.- P.166-172.

3. Кириченко Ю.В. Плазменная антенна бегущей волны / Кириченко Ю.В., Лонин Ю.Ф., Онищенко И.Н. //
Известия вузов. Радиоэлектроника. - 2011. - Т. 54, №. 11. - C.35-39.

4. Кириченко Ю.В. Плазменная антенна осевого излучения /Кириченко Ю.В., Лонин Ю.Ф., Онищенко И.Н.// Радиотехника и электроника.-2014.-m.59,№.3.- C.1-7.

5. М.В. Кузелев. Плазменная релятивистская электроника / М.В. Кузелев., А.А. Рухадзе, П.С. Стрелков.М.:МГТУ,2002, - 542 c.

6. Уолтер К. Антенны бегущеей волны / Уолтер К. М.: Энергия, 1970,- 448 c.

7. Особливості використання плоского шару плазми 3 малим вигином в антенних системах / В.Д. Карлов, Ю.В. Кириченко, А.С. Кійко // Збірник наукових прачьь Харківського начіонального університету Повітряних Сил. Х. : ХНУПС. - 2017. - Вип. 4(53). - C.97- 102.

8. Кириченко Ю.В. СВЧ излучение иилиндрического плазменного столба /Кириченко Ю.В., Лонин Ю.Ф., Онищенко И.Н.// Известия вузов. Радиоэлектроника. - 2014.m.547, №.10. - C.54-59.

9. Карлов В. Д. Характеристики эхо-сигналов декаметрового диапазона радиоволн при их многолучевом распространении / В. Д. Карлов, Ю. А. Сирык, А. В. Тугай // Системи озбросння $і$ військова техніка. - Х. - ХУПС. 2011. - №. 2(26). - C. 134-137.

10. Jenn D.C. Plasma antennas: Survey of Techniques and the Current State of the Art. Naval Postgraduate School, Prepared for SPAWWAR PMW 189. San Diego. 2003. 27 p.

11. Rayner J.P., Whichello A.P, Cheetham A.D. 2004, 'Physical characteristics of plasma antennas' IEEE Transaction on plasma science.32, P. 269-281.

12. Borg G.G., Harris J.H., Martin N.M., Thorncraft D. Plasmas as antennas: theory, experiment and application. Physics of Plasmas, V.7. №5, pp. 2198-2202, 2000.

Надійшла до редколегії 12.02.2018

Рецензент: д-р техн. наук, проф. Л.Ф. Купченко, Харківський національний університет Повітряних Сил імені Івана Кожедуба, Харків.

\title{
ОСОБЛИВОСТІ ФОРМУВАННЯ ДІАГРАМ СПРЯМОВАНОСТІ ПЛАЗМОВОЇ АНТЕНИ 3 АЗИМУТНО-НЕСИМЕТРИЧНИМИ ХВИЛЯМИ СТРУМУ, ЩО БІЖАТЬ
}

\author{
В.Д. Карлов, Ю.В. Кириченко, А.М. Артеменко, О.В. Бесова
}

Теоретично та чисельно досліджуться плазмова антена з азимутально-несиметричною хвилею, щуо біжить, яка працює на гармоніці з азимутним номером $m=1$. Плазма, в якій враховані електрон атомні зіткнення, передбачається холодною та ізотропною. Досліджується залежність діаграм спрямованості від щільності плазми, геометричних параметрів плазмового циліндра і частоти електрон атомних зіткнень. Коефіиієнти згасання, обумовлений зіткненнями, знаходяться з дисперсійного рівняння. Знайдено мінімальні значення щільності плазми, при яких нормовані діаграми спрямованості є гостронаправленою і мають головну пелюстку, яка розташована під нульовим кутом до осі антени. Показано переваги антени з несиметричною хвилею з $m=1$ в порівнянні з антеною, щзо працює на азимутальносиметричній моді.

Ключові слова: плазмова антена хвилі, що біжить, антена з випромінюванням вісі, лінійна антена, обмежена плазма, несиметричні поверхневі хвилі в плазмі, електроно атомні зіткнення в плазмі, діаграма спрямованості.

\section{FEATURES OF FORMING OF DIAGRAMS OF ORIENTATION OF PLASMA AERIAL WITH AZIMUTHAL-ASYMMETRICAL PROGRESSING WAVES OF CURRENT}

V. D. Karlov, Yu.V. Kirichenko, A. N. Artemenko, O. V. Besova

Theoretically and numerically, a plasma antenna with an azimuth-asymmetric traveling wave working on a harmonic with an azimuth number $m=1$ is studied. Plasma, in which electron-atom collisions are taken into account, is assumed to be cold and isotropic. The dependence of the directivity diagrams on the plasma density, the geometric parameters of the plasma cylinder, and the frequency of electron collisions are investigated. The attenuation coefficients due to collisions are found from the dispersion equation. The minimum values of the plasma density are found at which the normalized radiation patterns are highly directional and have a main lobe located at a zero angle to the antenna axis. The advantages of an antenna with an asymmetric wave with $m=1$ are shown in comparison with an antenna operating in an azimuthally symmetric mode.

Keywords: traveling wave plasma antenna, antenna of axial radiation, linear antenna, limited plasma, asymmetric surface waves in plasma, electron atomic collisions in plasma, directional diagram. 